\title{
Digit Ratio Predicts Spatial Memory Performance in Women
}

\author{
Lili Tcheang $^{1^{*}}$, Neil G. Muggleton ${ }^{1,2}$, Vincent Walsh ${ }^{1}$ \\ ${ }^{1}$ University College London, Institute of Cognitive Neuroscience, London, England \\ ${ }^{2}$ National Central University, Institute of Cognitive Neuroscience, Jhongli City, China \\ Email: ${ }^{1}$ tcheang@gmail.com
}

Received April 2, 2013; revised May 10, 2013; accepted May 22, 2013

Copyright (C) 2013 Lili Tcheang et al. This is an open access article distributed under the Creative Commons Attribution License, which permits unrestricted use, distribution, and reproduction in any medium, provided the original work is properly cited.

\begin{abstract}
The ratio of the length of the 2 nd and 4th digits of the hand is an indicator of prenatal testosterone exposure and has been shown to be a within sex correlate of various abilities, such as visuo-spatial processing in men. However, a number of studies report such links also exist in women, whilst others show no link for either sex. Using a unique paradigm that sub-divides visuo-spatial processing into a distinct subset of component skills we found a strong correlation between finger ratio and spatial memory performance specifically under stereo viewing conditions in women. We argue that the current ambiguity regarding links between digit-ratio and visuo-spatial ability is the result of a lack of clarity between the component skills recruited in different visuo-spatial tasks. Our task independently tested a subset of the component skills used in visuo-spatial tasks such as: mental rotation, cross-dimensionality processing and feature detection. Our results show that digit-ratio, a physical parameter linked to prenatal testosterone levels, indicates performance on a distinct aspect of visuo-spatial processing in women, without contradicting previous links for visuo-spatial processing in men. These results offer an explanation for the differences in previous findings linking visuo-spatial processing in both men and women.
\end{abstract}

Keywords: Digit Ratio; Visuo-Spatial Memory; Spatial Memory; Mental Rotation; Sex Differences

\section{Introduction}

\subsection{Digit Ratio and Visuo-Spatial Performance}

Digit ratio, in particular the ratio of the length of the 2 nd finger to the 4th finger, has been found in humans to be a correlate of the level of individual prenatal testosterone exposure [1]. Typically, men tend to have lower digit ratios as a result of being exposed to higher testosterone levels in the womb $[2,3]$. Male digit ratio has been correlated with a range of abilities, including those as diverse as visuo-spatial processing [4,5] and success in financial trading [6]. At the same time, the digit ratio in females has been linked to a number of personality traits, such as psychoticism and neuroticism [7]. Together these studies suggest a difference in the way prenatal testosterone levels influence male and female behaviour. However, this distinction is not so clear-cut with studies showing a link between female digit ratio and spatial navigation ability [8] as well as the sense of direction [9], another finding shows links for both sexes for a line angle judgement

${ }^{*}$ Corresponding author. task [10], with further studies including large samples, showing no links between either sexes' digit ratio and cognitive task performance [3,7], and finally a meta analysis reiterating these conclusions [11].

\subsection{Verbal Ability, Testosterone and Visuo-Spatial Performance}

The literature linking testosterone levels and spatial performance is complicated by reports of both negative and positive correlations between testosterone levels and visuo-spatial task performance for men and women respectively. These studies postulate an inverted u-shape function with an optimal level of testosterone linked to maximal visuo-spatial performance $[12,13]$. This theory is consistent with the existence of a positive correlation between salivary testosterone levels and visuo-spatial task performance for women, whose testosterone levels tend to be along the lower end of the scale, whilst at the same time, explaining a negative correlation between salivary testosterone levels and visuo-spatial performance for men, who tend to have higher testosterone levels. 
A study in androgen deficient men showing a positive correlation between testicular volume and spatial ability provides evidence in favour of this theory [14]. Further support exists in the sex differences literature with males performing slightly better on visuo-spatial working memory tasks, whilst females perform better on verbal fluency tasks [15-18]. The dichotomy of task type and performance suggests a trade-off between verbal and visuospatial abilities irrespective of sex differences.

\subsection{Handedness and Visuo-Spatial Performance}

Handedness, like digit ratio, has also been shown to be an indicator of prenatal exposure to testosterone. In humans, the fact that the right hemisphere of the brain develops faster than the left, coupled with the theory that testosterone exposure inhibits the growth of neurons, may indicate that more testosterone can slow the growth of the left hemisphere and result in cognitive differences [19]. These differences include a higher incidence of lefthandedness in right hemisphere dominant brains. Consequently, because males in general have higher levels of prenatal testosterone, there are more incidences of lefthandedness for males than there are for females [20-22]. The retention of visual material has been linked to handedness [23] and there is also evidence to suggest a similar relationship between handedness and spatial ability, similar to the proposed link between prenatal testosterone levels and spatial ability, where lower spatial performance was linked with extreme right or left handedness, whilst highest performers fell somewhere in between [24]. Therefore, the link between handedness and visuospatial performance lends weight to the suggestion that digit ratio is linked to performance on visuo-spatial tasks, with both being a consequence of prenatal testosterone exposure.

\subsection{D Technology and Visuo-Spatial Performance}

With the advent of 3D screen technology entering mainstream media entertainment, there are many questions as to how this might enhance viewers' experiences. In a research context, this technology allows for easier investigation of, for example, whether the addition of stereo information increases the ability to process the rotation of visual information (mental rotation). One paper has reported that stereo information is only useful for very specific three dimensional tasks, such as for air traffic controllers having to work on a collision avoidance task [25]. Postulated sex differences were not confirmed on mental rotation tasks when real objects were used or when a 3D stereo rendition of objects was used [26,27]. The addition of 3D information appears to simplify the task of mental rotation by eliminating the need to infer the $3 \mathrm{D}$ structure of a 2D image, a process known as dimensionality crossing [28]. It therefore appears that males have an advantage in conventional mental rotation tasks because of their ability to extract 3D information from a perspective projection, rather than in mental rotation per se.

\subsection{Aspects of Visuo-Spatial Ability}

Visuo-spatial processing involves a number of skills that could explain the variety of conclusions across existing studies linking it to digit ratio including: mental rotation, cross-dimensionality processing and detection of spatial feature changes. Whilst a number of studies have shown distinct differences in performance on different aspects of visuo-spatial processing between the sexes, digit ratio links to visuo-spatial ability consist of a number of contradictory studies to date. In the sex differences literature, men tend to perform better on visuo-spatial tasks that primarily involve mental rotation[16,27,29] or extracting $3 \mathrm{D}$ spatial information from $2 \mathrm{D}$ visual information [27, 30]. Whereas women often outperform men on tasks that favour object location memory $[31,32]$. It is therefore important to separately investigate these aspects of visuospatial ability, in order to investigate the role of digit ratio in visuo-spatial performance. For example, if male ability on mental rotation tasks is a result of prenatal testosterone exposure, this effect would be diluted in a study that used a visuo-spatial task that did not separate this aspect from feature detection or mental rotation ability. We employed a novel paradigm that enabled us to study the relative effects of the different skills involved during visuo-spatial processing. Our stimuli were based on an existing spatial memory task where participants were required to judge whether a presented landscape showed the same place as a subsquently presented landscape [33]. Landscapes used consisted of four mountains in a valley which could change in a combination of two ways: 1) the viewpoint from which the landscape is viewed is changed while the landscape itself is unchanged, allowing examination of mental rotation skills in isolation from other visuo-spatial processing skills; 2) spatial features in the landscape are changed, such as the size, shape or position of mountains. This allows examination of the detection of spatial changes, again in isolation from other visuo-spatial processing skills. In addition to this, separate sessions were employed in which the stimuli were presented in a $2 \mathrm{D}$ perspective view or viewed in $3 \mathrm{D}$ by means of stereo liquid crystal shutter glasses. This allowed us to examine dimensionality crossing in isolation from other visuo-spatial processing skills.

\section{Material and Methods}

All participants gave written, informed consent prior to 
taking part in the experiment and were compensated for their participation. The study was approved by the ethics committee of the Department of Psychology at UCL.

\subsection{Digit Ratio}

Digit ratio was measured by photocopying the hands of participants using the same method as Coates, et al. [6], where finger lengths were measured manually with a ruler.

\subsection{Equipment}

Stimuli were presented on a 22 inch Samsung SM2233RZ monitor with a refresh rate of $120 \mathrm{~Hz}$ which was viewed from a distance of $57 \mathrm{~cm}$. Participants wore shutter glasses synced to the frequency of the monitor, such that each eye was exposed to alternate frames of the display, allowing presentation of stereo images at an effective frequency of $60 \mathrm{~Hz}$. The experiment was coded in $\mathrm{C} \# / \mathrm{XNA}$ running on Windows 7.

\subsection{Participants}

30 right-handed participants, 14 females and 16 males took part in the experiment. Mean age was 26.00 with a standard deviation 5.20 years. Data for 1 male participant was excluded on the grounds that his score on the spatial memory task was less than chance.

\subsection{Verbal Fluency Test}

Participants conducted a semantic fluency test chosen as it has been shown to be most transferable across languages [34]. Participants had the choice of performing the test in their native language or in English, as well as whether to conduct it in a word processing document or to write the list out manually. Participants had one minute to list as many animals as they could.

\subsection{Handedness Questionnaire}

Participants filled in a modified version of the original Edinburgh handedness questionnaire [35]. http://www.brainmapping.org/shared/Edinburgh.php

\subsection{Ravens Progressive Matrices Test}

To control for the effects of IQ on visuo-spatial performance, participants performed the 12 -item version of the Raven's Matrices (Set 2). This version of the test gives a reasonable indication of IQ within a short time frame $[15,36]$.

\subsection{Visuo-Spatial Task: 4 Mountains Task}

The visuo-spatial task was presented in 4 separate sessions, each consisting of 24 blocks of 5 images. 2 ses- sions involved presentation of stimuli in $2 \mathrm{D}$, whilst 2 sessions involved presentation of stimuli in 3D. The order was randomised across different participants. Stimuli were adapted from Hartley, et al. [33]. Each image consisted of a landscape with four mountains of different shapes and sizes. For the first image in each block, participants only had to memorise the landscape and its particular arrangement of features. Participants then advanced to the next image by pressing either the number 1 or 2 on the keyboard numerical keypad. In the subsequent 4 images the landscape changed in one of four ways relative to the preceding image in a pseudo-random order:

1) The landscape and viewpoint from which it was seen stayed the same.

2) Spatial features such as two of the mountains changed shape or size in the landscape.

3) The landscape remained the same, but the viewpoint changed.

4) Both the spatial features and the viewpoint of the new landscape also changed.

Participants were required to judge whether an image was the same or a different place as the preceding image using the "1" key for locations they thought were the same (conditions 1 and 3) and the " 2 " key if they thought they were different (conditions 2 and 4). Figure 1 shows examples of the type of stimuli presented. There was no time limit for responding and accuracy was emphasised. No feedback was provided but participants were given a demonstration of one block of trials before commencing the experiment to familiarise themselves with the task.

\section{Results}

The main result was a link between female digit ratio and visuo-spatial performance as a function of the type of visual display.

\subsection{General Performance}

Performance did not differ significantly between men and women (unpaired $t$-test $t(28)=0.0355, p=0.97$ ) with mean of 280.27 correct trials out of a total of 384 trials, even when separated into the four change conditions (i.e. when no changes occur between images, unpaired $t$-test $t(28)=0.5947, p=0.56$; when a spatial change occurs, unpaired $t$-test $t(28)=0.7103, p=0.48$; when a viewpoint change occurs, unpaired $t$-test $t(28)=$ $0.2390, p=0.81$ and when both a viewpoint and spatial change occurs $t(28)=0.1379, p=0.89$ ).

Pooling male and female data together, there was a significant difference between 2D and 3D presentations for the spatial change only condition; scenario 2 in Figure 1A (paired $t(29)=2.39, p=0.0234, r=0.41$ ), with performance being worse when the stimuli were 


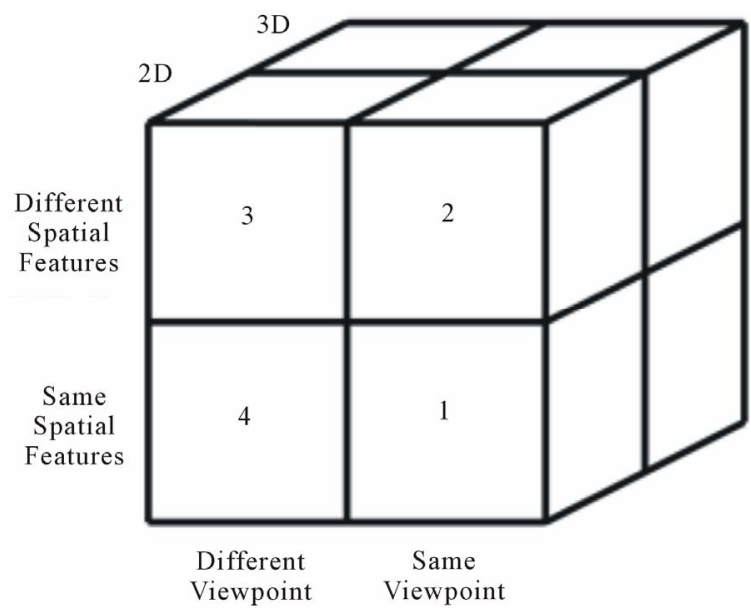

(a)

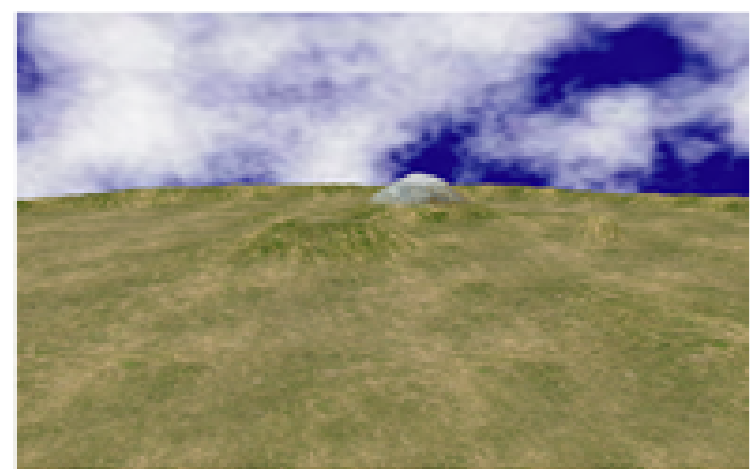

(b)
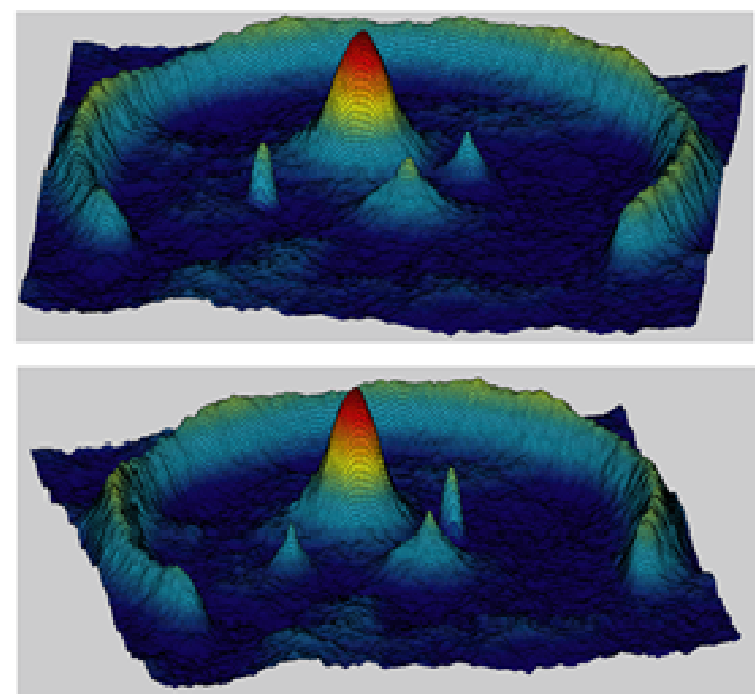

(c)

Figure 1. (a) Schematic summarizing the 8 possible types of stimuli presented. The numbers on the cube faces represent the four possible ways the two changes between images combine, with the depth of the cubes representing presentations shown in 2D or 3D. (b) Illustration of an image seen by participants. (c) Schematic showing one possible combination of changes. In this case, both a spatial feature change has occurred, and a viewpoint change (condition number 4 in Figure 1(a)). presented in 3D as opposed to 2D (30.9 c.f. 32.8).

\subsection{Sex Differences in the Independent Measures}

Figure 2 shows differences between the sexes on the independent predictors of visuo-spatial ability. In general there were no significant differences between the measures with respect to sex with the exception of digit ratio, which in agreement with past studies, showed that male digit ratios were significantly lower than female digit ratios (unpaired $t(28)=3.62, p=0.005$, Bonferroni corrected, $r=0.5647)$.

\subsection{Independent Measures Correlations}

Cross-correlations between the predictors: age, Raven's matrices scores, verbal fluency, handedness and digit ratio showed no significant correlations for either men or women.

\subsection{Regression Analysis}

\subsubsection{Overall Data}

Simple linear regression showed significant correlations in the overall data between age and the following conditions:

1) Age and detection of no changes $(r=0.39, p=$ $0.0347)$.

2) Age and detection of no changes in 3D $(r=0.42, p$ $=0.0212$ ).

3) Age and detection of viewpoint changes in 3D $(r=$ $0.39, p=0.0348)$.

4) Digit ratio and detection of a spatial change in $3 \mathrm{D}(r$ $=-0.42, p=0.0211$ ).

The first two correlations were discounted, since detection of no changes does not necessarily reflect spatial ability.
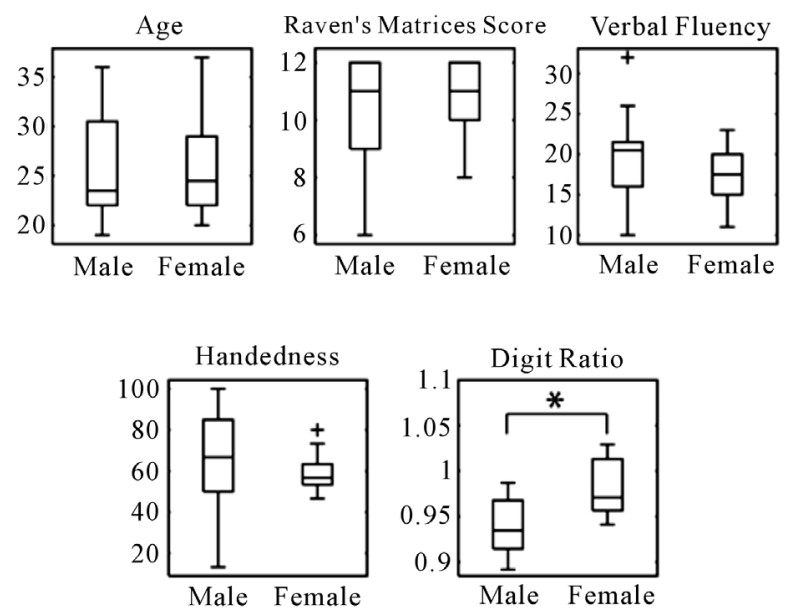

Figure 2. Distribution of independent predictors of visuospatial ability. Unpaired $t$-tests showed a significant sex difference only for the digit ratio. "shows significant unpaired $t$-test, $p=0.0011$. 
The third correlation between age and viewpoint change is opposite to many in the previous literature that show a negative link between age and visuo-spatial ability [37-40]. However in these studies the older age group was typically $>60$, whilst in our study, the youngest participant was 19 whilst the oldest was 37 .

The correlation between digit ratio and the detection of a spatial change in 3D mirrors the correlation for the female data alone (see below) but it is not present in the male data $(r=0.2386, p=0.3735)$ so can be attributed to the effects of the female data carrying over. Again similar to the female only data, the detection of no changes, which is not strictly a visuo-spatial task, showed a significant correlation.

\subsubsection{Male Data}

Simple linear regression showed no significant correlations in the male data. However, there were two correlations close to significance:

1) Ravens Matrices score and detection of a spatial change $(r=0.46, p=0.0640)$.

2) Ravens Matices score and detection of a spatial change in $2 \mathrm{D}(r=0.50, p=0.0503)$.

No other correlation resulted in a $\mathrm{p}$ value less than 0.1 . The near-significant correlations with Ravens matrices score mirror a large sample study $(n=280)$ that showed a link between Ravens Matrices score and visuospatial abilities [36].

\subsubsection{Female Data}

In the female data, the following positive correlations were seen:

1) Digit ratio and the spatial task only when the stimuli were in 3D and a spatial change had occurred $(r=-0.70$, $p=0.0057$ ), see also the top row of Figure 3 .

2) Digit ratio and the spatial task in $3 \mathrm{D}$ when a viewpoint and spatial change had occurred $(r=-0.77, p=$ $0.00141)$.

3) Handedness and overall performance in both $2 \mathrm{D}$ and $3 \mathrm{D}$ versions of the task $(r=0.76, p=0.0017$ and $r=$ $0.74, p=0.0023$ respectively).

4) Handedness and detection of a spatial change in the task in both $2 \mathrm{D}$ and $3 \mathrm{D}(r=0.54, p=0.0486$ and $r=0.58$, $p=0.0302$ respectively).

5) Age and detection of no changes between stimuli when viewed in $3 \mathrm{D}(r=0.55, p=0.0421)$.

Figure 3 summarises the significant correlations for the female data.

No correlation between digit ratio and detection of viewpoint change alone in $3 \mathrm{D}$ was found $(r=0.19, p=$ $0.5071)$. This suggests that the correlation between Digit Ratio and detection of changes when both viewpoint and spatial features change (see Correlation 2 and Figure 3) does not depend on the viewpoint change and reflects the correlation in the detection of a spatial change alone.

As there were multiple predictors for the detection of spatial change in $3 \mathrm{D}$, a hierarchical multiple regression was performed on the female data, with the digit ratio entered as the main predictor followed by Handedness, then Verbal Fluency, Ravens Matrices Score and Age together. The order for the predictors was selected using criteria described in Field [41]. In the simple regression, digit ratio alone accounted for $44.1 \%$ of the variability in detecting a spatial change in 3D stimuli and an associated significant F-ratio $(r=-0.70, p=0.006)$. Handedness accounted for another $20.2 \%$ with an associated significant F-ratio (multiple $r=0.828, p=0.022$ ). The addition of verbal fluency, Raven's Matrices Score and age did not have an associated significant F-ratio $(p=0.146)$, so their contribution from the regression can be excluded. Compared with $r$ square and multiple $r$ square, adjusted $r$ square and adjusted multiple $r$ square calculated from Wherry's equation, are of a similar size ( $r$ square: adjusted $r$ square, 0.484:0.441 and multiple $r$ square: adjusted multiple $r$ square, 0.686:0.629). This suggests that the sample female data is a good representation of the population that the sample originates from. Given that both digit ratio and handedness showed significant correlations with the detection of a spatial change in $3 \mathrm{D}$, the regression between digit ratio and handedness was assessed to examine whether they could be treated as independent predictors. The correlation was not significant: $r$ $=0.20, p=0.4978$.

These results suggest that whenever $3 \mathrm{D}$ spatial features change in a scene, detection of these changes correlates strongly with $2 \mathrm{D}: 4 \mathrm{D}$ ratio and handedness in women as independent predictors.

\subsubsection{Post Hoc $t$-Tests}

Given the correlation for females between digit ratio and performance in the 3D spatial change condition, a unpaired $t$-test between male and female performance in the spatial change condition in 3D was performed. No significant difference was seen $(t(28)=0.371, p=0.71)$.

\section{Discussion}

\subsection{Digit Ratio and Visuo-Spatial Performance}

The negative correlation between $3 \mathrm{D}$ stereo spatial change detection and digit ratio for women found in the present study is a new finding and gives insight into the seemingly conflicting studies that show a correlation between spatial ability and digit ratio in either women only [8,42], men only [4], in both men and women [5], [10] or in neither $[3,7,18]$. Most importantly this negative correlation, suggesting higher prenatal testosterone exposure in women confers an advantage in spatial feature memory, agrees with existing studies showing that visuo- 


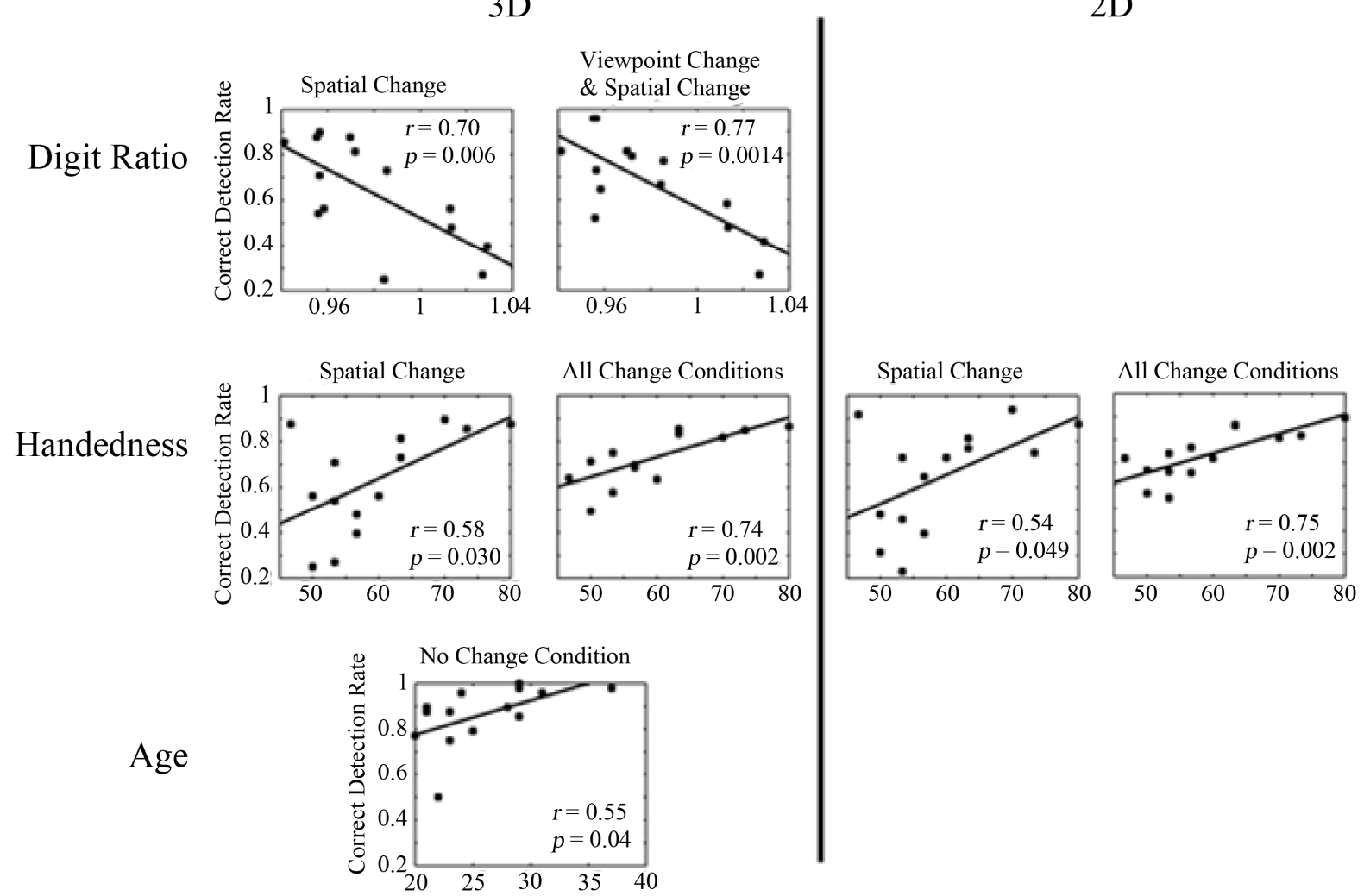

Figure 3. Significant correlations in 3D on the left and 2D on the right. Rows separate different predictor variables: Digit Ratio describes the ratio of the length of the second to the fourth digit, Handedness measures the level of handedness between -100 to 100 where -100 is fully left-handed and 100 is fully right-handed and age is given in years. Graph titles describe the type of visual change between consecutive stimuli.

spatial performance correlates with higher testosterone levels for women $[12,16]$. It is also consistent with large sample internet studies ( $>250,000$ subjects) showing that spatial ability correlated with more male-typical digit ratios $[5,10]$.

A key difference between our task and visuospatial tasks typically employed in previous studies such as the previously mentioned Sanders, et al. [4] study, which showed only correlations with male digit ratio, is that the conventional Shepard and Metzler task they used requires explicit dimensionality crossing, whereby the observer must extract $3 \mathrm{D}$ information from a $2 \mathrm{D}$ perspective view, and perform mental rotation on every trial. It has been shown that men perform better on mental rotation tasks although this advantage disappears when dimensionality crossing is eliminated from the task [27]. Analogously, the link between female digit ratio and spatial feature detection is similar to the female advantage in perceptual speed judgements of identifying similar pictures in a group of others [12]. It also supports findings of a female advantage in object location memory [31,32]. The findings in the current study therefore mirror the sex differences seen in tasks which may be appropriately comparable.
In our study, mental rotation was only necessary in cases where the viewpoint changed. We found that in both this condition and the one in which the viewpoint stayed the same, performance correlated with digit ratio when only spatial features changed, and only when the task was in 3D. More specifically, there was a correlation between female digit ratio and a specific spatial memory skill in $3 \mathrm{D}$ in the absence of both mental rotation and dimensionality crossing.

We argue that the reason for sex differences previously reported lies in the different types of visuo-spatial task that participants were required to perform. With regards to our own data, a pure spatial feature change detection task in the absence of mental rotation and dimensionality crossing was the specific aspect of the task that showed a correlation with female digit ratio.

We also suggest that the stimuli we presented were a novel stimulus type that participants would not ordinarily be exposed to, and could explain the strong correlations in our data. At first glance, the idea that viewing and processing 3D stimuli is novel seems contradictory, given that we experience the natural world in stereo. However, one indication of task novelty in our results is the lower performance on the task when presented in 3D 
compared with in $2 \mathrm{D}$. This decrease in performance for 3D stimuli is contrary to other studies that have shown that $3 \mathrm{D}$ information can enhance performance on visuospatial processing tasks with the caveat that the task involves a particular aspect of spatial processing $[25,43]$. In addition, one study makes a distinction between 3D information shown in a $2 \mathrm{D}$ perspective view on a screen, 3D non-immersive (using anaglyphic glasses) viewing and 3D immersive viewing, and argues that 3D nonimmersive viewing does not differ significantly from 2D viewing, whilst both are inferior to $3 \mathrm{D}$ immersive viewing [43]. The significant difference between $2 \mathrm{D}$ and $3 \mathrm{D}$ performance, coupled with the correlation between female digit ratio and performance in $3 \mathrm{D}$ in our study adds to this finding and shows that desktop 3D is qualitatively different from desktop 2D. This may be due to there being subtle differences between stereo information viewed in a natural scene and stereo information viewed on a stereo display. To elaborate on this, a landscape viewed in the real world would not possess the same large disparity information that is used to represent the landscape in stereo on a small desktop display. In fact the desktop 3D information may be quite different compared to the same sort of scene in the real environment, since large and distant landscapes would lack the level of disparity information artificially introduced here. Indeed a 2D landscape could be argued to be a closer rendition of a real landscape viewed from afar, as the real landscape would possess very little stereo information. Additionally, we are very used to organising $2 \mathrm{D}$ images into $3 \mathrm{D}$ landscapes, having for most of our lives been exposed to 2D images of 3D scenes from static pictures to moving images. It is therefore possible that despite viewing the natural world in 3D, the lack of familiarity in viewing artificial visual stimuli encoded with 3D information gives an advantage to the normal process of reconstructing $3 \mathrm{D}$ structure from the very commonplace $2 \mathrm{D}$ stimuli. The addition of stereo in this case could be viewed as rather artificial. In fact, one could argue that the $3 \mathrm{D}$ information provided in this experiment was more representative of a small-scale model landscape and therefore represents an artificial and relatively novel rendition of 3D. As such, by presenting the stimuli in 3D we may have effectively introduced a novel paradigm that participants have not experienced before, reducing practice effects as a result of lifetime exposure to such tasks, which could explain why such a strong correlation was found for the female data. In time, with the possible advent of 3D stimuli becoming more mainstream, processing of such scenes may become second nature and reach the same levels that $2 \mathrm{D}$ scenes are currently processed with. A side-effect of this might be the reduction of the strength of the correlation with female digit ratio as different levels of familiarity with the stimuli confound any intrinsic spatial processing skills.

The distinction between spatial ability and mental rotation ability, and the absence of cross-dimensionality processing, offers an explanation to the existing inconsistency in the literature regarding sex differences and links to the digit ratio. Also, because female testosterone levels are typically much lower than males, their innate visuo-spatial abilities are more closely linked to prenatal testosterone levels, with the latter being a marker of instinctive performance. In males, innate visuo-spatial ability is likely to be influenced by their much higher ongoing testosterone levels, although it is important to note that this does not negate the findings of the previous study that showed a correlation between male performance on a visuo-spatial task and 2D:4D ratio [4]. In contrast to our experimental task, their task was based on more correctly scaled objects [44], was presented in a more familiar 2D display setting and imposed a time limit in which participants could respond. Other such correlations in the male data have also been found but with much larger sample sizes $(>100,000)$ than the ones we have utilised here [5]. It is therefore possible that with a larger sample size, a correlation with male digit ratio and spatial performance in our task would also be found. Nevertheless, this study did not find a correlation with an equivalent-sized sample of males to females, with the latter showing significant effects.

\subsection{Handedness and Visuo-Spatial Performance}

The handedness correlation found for performance in women is consistent with previous studies showing a correlation with right handedness and spatial ability in women $[45,46]$. The present study shows the correlation for a much smaller sample size (14 compared with more than 100) and is compelling given the narrower spread in handedness for females compared with males (see Figure 2). Interestingly, the correlations exist for both the 2D and 3D data, with $p$ values an order of magnitude lower when the viewpoint also changed and when participants responded correctly to detecting no changes between subsequent images (see Figure 3). It therefore appears that the correlation with handedness is distinct from the correlation with digit ratio, with handedness being a predictor of general visuo-spatial processing performance, irrespective of whether the task is shown in $2 \mathrm{D}$ or 3D. Handedness is therefore not subject to the same novelty conditions that the digit ratio correlation seems to be. Additionally, we did not find a correlation between handedness and digit ratio, in contrast to previous studies. This is less surprising in light of the evidence above showing differences between how handedness and digit ratio relate to different aspects of the spatial stimuli. This result adds evidence to the theory that prenatal testosterone is the primary independent variable that gives 
rise to handedness and digit ratio, which in turn are linked to different aspects of spatial processing separately. However, handedness studies again tend to have much larger sample sizes and it is likely that the association between handedness and digit ratio is therefore much weaker $[47,48]$.

\subsection{Verbal Fluency}

Given the dichotomy between the sexes for verbal and spatial abilities $[15,16]$, we investigated whether the two were negatively correlated. We found no correlation between spatial ability and verbal fluency, suggesting that verbal fluency does not predict spatial ability. We also did not find a significant sex difference (see Figure 2). This finding is consistent with the current literature where some studies show a difference [16,29] and others show no sex differences in verbal ability [49]. This finding however, does not impact on our main finding, that there is a link between spatial performance and digit ratio for female participants.

\subsection{Age}

The positive correlation of age with one aspect of the spatial memory task contrasts with a number of existing studies showing a deficit in performance with age [37,50, 51]. However, the particular aspect of the scene that correlated with age was detection of "no changes". Detection of the image remaining exactly the same is not strictly speaking a spatial processing task so it is not an equivalent comparison to past studies. When the viewpoint of the scene changed or when spatial aspects of the scene changed, performance in these tasks was not found to be age related in contrast to previous studies.

\section{Conclusion}

By comparing the correlations between the digit ratio and specific stimuli conditions, we have shown that digit ratio is a strong predictor of the detection of spatial changes during visuo-spatial processing in 3D stimuli for women but not for men. The correlation for women is evident even in the absence of a mental rotation step incorporated as a part of the visuo-spatial task. This finding adds clarity to existing studies showing a link between digit ratio in men and visuo-spatial ability, where mental rotation and cross-dimensionality processing often formed a key part of the visuo-spatial task. Our findings shed light on how specific component skills that fall under the umbrella of visuo-spatial processing are linked to the digit ratio in women and men separately.

\section{Acknowledgements}

The authors thank Christina Tsakali for her assistance with data collection. This work was funded by the MRC, grant number G0700929, www.mrc.ac.uk. NGM was also funded by the National Science Council, Taiwan (N.G.M.; 100-2410-H-008-074-MY3).

\section{REFERENCES}

[1] S. A. Berenbaum, K. K. Bryk, N. Nowak, C. A. Quigley and S. Moffat, "Fingers as a Marker of Prenatal Androgen Exposure," Endocrinology, Vol. 150, No. 11, 2009, pp. 5119-5124. doi:10.1210/en.2009-0774

[2] J. Manning, "Digit Ratio: A Pointer to Fertility, Behavior, and Health," Rutgers University Press, New Brunswick, 2002, p. 173.

[3] J. Coolican and M. Peters, "Sexual Dimorphism in the 2D/4D Ratio and Its Relation to Mental Rotation Performance," Evolution and Human Behavior, Vol. 24, No. 3, 2003, pp. 179-183. doi:10.1016/S1090-5138(03)00010-2

[4] G. Sanders, T. Bereczkei, A. Csatho and J. Manning, "The Ratio of the 2nd to 4th Finger Length Predicts Spatialability in Men but Not Women," Cortex, Vol. 41, No. 6, 2005, pp. 789-795. doi:10.1016/S0010-9452(08)70297-1

[5] M. Peters, J. T. Manning and S. Reimers, "The Effects of Sex, Sexual Orientation, and Digit Ratio (2D:4D) on Mental Rotation Performance," Archives of Sexual Behavior, Vol. 36, No. 2, 2007, pp. 251-260. doi:10.1007/s10508-006-9166-8

[6] J. M. Coates, M. Gurnell and A. Rustichini, "Second-toFourth Digit Ratio Predicts Success among High-Frequency Financial Traders," Proceedings of the National Academy of Sciences of the United States of America, Vol. 106, No. 2, 2009, pp. 623-8.

doi:10.1073/pnas.0810907106

[7] E. Austin, J. Manning, K. Mcinroy and E. Mathews, “A Preliminary Investigation of the Associations between Personality, Cognitive Ability and Digit Ratio," Personality and Individual Differences, Vol. 33, No. 7, 2002, pp. 1115-1124. doi:10.1016/S0191-8869(02)00002-8

[8] A. Csathó, "Spatial Navigation Related to the Ratio of Second to Fourth Digit Length in Women," Learning and Individual Differences, Vol. 13, No. 3, 2003, pp. 239-249. doi:10.1016/S1041-6080(02)00093-6

[9] X. J. Chai and L. F. Jacobs, "Digit Ratio Predicts Sense of Direction in Women," PLoS One, Vol. 7, No. 2, 2012 , p. e32816. doi:10.1371/journal.pone.0032816

[10] M. L. Collaer, S. Reimers and J. T. Manning, "Visuospatial Performance on an Internet Line Judgment Task and Potential Hormonal Markers: Sex, Sexual Orientation, and 2D:4D," Archives of Sexual Behavior, Vol. 36, No. 2, 2007, pp. 177-92. doi:10.1007/s10508-006-9152-1

[11] D. Puts, M. McDaniel, C. L. Jordan and S. M. Breedlove, "Spatial Ability and Prenatal Androgens: Meta-Analyses of Congenital Adrenal Hyperplasia and Digit Ratio (2D:4D) Studies," Archives of Sexual Behavior, Vol. 37, No. 1, 2008, pp. 100-111. doi:10.1007/s10508-007-9271-3 
[12] C. Gouchie and D. Kimura, "The Relationship between Testosterone Levels and Cognitive Ability Patterns," Psychoneuroendocrinology, Vol. 16, No. 4, 1991, pp. 323334. doi:10.1016/0306-4530(91)90018-O

[13] S. D. Moffat and E. Hampson, "A Curvilinear Relationship between Testosterone and Spatial Cognition in Humans: Possible Influence of Hand Preference," Psychoneuroendocrinology, Vol. 21, No. 3, 1996, pp. 323-337. doi:10.1016/0306-4530(95)00051-8

[14] D. B. Hier and W. F. Crowley, "Spatial Ability in Androgen-Deficient Men," The New England Journal of Medicine, Vol. 306, No. 20, 1982, pp. 1202-1205.

[15] D. F. Halpern and M. L. Lamay, "The Smarter Sex: A Critical Review of Sex Differences in Intelligence," Educational Psychology Review, Vol. 12, No. 2, 2000, pp. 229-246. doi:10.1023/A:1009027516424

[16] L. A. Burton, D. Henninger and J. Hafetz, "Gender Differences in Relations of Mental Rotation, Verbal Fluency, and SAT Scores to Finger Length Ratios as Hormonal Indexes," Developmental Neuropsychology, Vol. 28, No. 1, 2005, pp. 493-505. doi:10.1207/s15326942dn2801 3

[17] J. M. Andreano and L. Cahill, "Sex Influences on the Neurobiology of Learning and Memory," Learning \& Memory, Vol. 16, No. 4, 2009, pp. 248-266. doi:10.1101/lm.918309

[18] N. Nowak and S. Moffat, "The Relationship between Second to Fourth Digit Ratio, Spatial Cognition, and Virtual Navigation," Archives of Sexual Behavior, Vol. 40, No. 3, 2011, pp. 575-585. doi:10.1007/s10508-010-9668-2

[19] N. Geschwind and A. M. Galaburda, "Cerebral Lateralization," MIT Press, Cambridge, 1987.

[20] M. P. Bryden, "Measuring Handedness with Questionnaires," Neuropsychologia, Vol. 15, No. 4-5, 1977, pp. 617624. doi:10.1016/0028-3932(77)90067-7

[21] C. Hardyck, R. Goldman and L. Petrinovich, "Handedness and Sex, Race, and Age," Human Biology, Vol. 47, No. 3, 1975, p. 369.

[22] C. Porac and S. Coren, Lateral Preferences and Human Behavior. Springer-Verlag, New York, 1981. doi:10.1007/978-1-4613-8139-6

[23] R. D. Nebes and G. G. Briggs, "Handedness and the Retention of Visual Material," Cortex, Vol. 10, No. 3, 1974, pp. 209-214. doi:10.1016/S0010-9452(74)80012-2

[24] S. A. Burnett, D. M. Lane and L. M. Dratt, "Spatial Ability and Handedness," Intelligence, Vol. 6, No. 1, 1982, pp. 57-68. doi:10.1016/0160-2896(82)90020-4

[25] K. Van Orden and J. W. Broyles, "Visuospatial Task Performance as a Function of Two- and Three-Dimensional Display Presentation Techniques," Displays, Vol. 21, No. 1, 2000, pp. 17-24. doi:10.1016/S0141-9382(00)00024-X

[26] W. McWilliams, C. J. Hamilton and S. J. Muncer, "On Mental Rotation in Three Dimensions," Perceptual and Motor Skills, Vol. 85, No. 1, 1997, pp. 297-298. doi:10.2466/pms.1997.85.1.297

[27] A. C. Neubauer, S. Bergner and M. Schatz, "Two- vs. Three-Dimensional Presentation of Mental Rotation
Tasks: Sex Differences and Effects of Training on Performance and Brain Activation," Intelligence, Vol. 38, No. 5, 2010, pp. 529-539. doi:10.1016/j.intell.2010.06.001

[28] P. Horan and R. Rosser, "A Multivariable Analysis of Spatial Abilities by Sex," Developmental Review, Vol. 4, No. 4, 1984, pp. 387-411. doi:10.1016/0273-2297(84)90023-6

[29] R. Halari, T. Sharma, M. Hines, C. Andrew, A. Simmons and V. Kumari, "Comparable fMRI Activity with Differential Behavioural Performance on Mental Rotation and Overt Verbal Fluency Tasks in Healthy Men and Women," Experimental Brain Research, Vol. 169, No. 1, 2006, pp. 1-14. doi:10.1007/s00221-005-0118-7

[30] D. Voyer, S. Voyer and M. P. Bryden, "Magnitude of Sex Differences in Spatial Abilities: A Meta-Analysis and Consideration of Critical Variables," Psychological Bulletin, Vol. 117, No. 2, 1995, pp. 250-70. doi:10.1037/0033-2909.117.2.250

[31] D. Voyer, A. Postma, B. Brake and J. Imperato-McGinley, "Gender Differences in Object Location Memory: A Meta-Analysis," Psychonomic Bulletin \& Review, Vol. 14, No. 1, 2007, pp. 23-38. doi:10.3758/BF03194024

[32] I. Ecuyer-Dab and M. Robert, "The Female Advantage in Object Location Memory According to the Foraging Hypothesis: A Critical Analysis," Human Nature, Vol. 18, No. 4, 2007, pp. 365-385. doi:10.1007/s12110-007-9022-0

[33] T. Hartley, C. M. Bird, D. Chan, L. Cipolotti, M. Husain, F. Vargha-khadem and N. Burgess, "The Hippocampus Is Required for Short-Term Topographical Memory in Humans," Hippocampus, Vol. 17, No. 1, 2007, pp. 34-48. doi:10.1002/hipo. 20240

[34] A. Ardila, F. Ostrosky-Solís and B. Bernal, "Cognitive Testing toward the Future: The Example of Semantic Verbal Fluency (ANIMALS)," International Journal of Psychology, Vol. 41, No. 5, 2006, pp. 324-332. doi:10.1080/00207590500345542

[35] R. C. Oldfield, "The Assessment and Analysis of Handedness: The Edinburgh Inventory," Neuropsychologia, Vol. 9, No. 1, 1971, pp. 97-113. doi:10.1016/0028-3932(71)90067-4

[36] K. Schweizer, F. Goldhammer, W. Rauch and H. Moosbrugger, "On the Validity of Raven's Matrices Test: Does Spatial Ability Contribute to Performance?" Personality and Individual Differences, Vol. 43, No. 8, 2007, pp. 1998-2010. doi:10.1016/i.paid.2007.06.008

[37] L. Jenkins, J. Myerson, J. A. Joerding and S. Hale, "Converging Evidence that Visuospatial Cognition Is More Age-Sensitive than Verbal Cognition," Psychology and Aging, Vol. 15, No. 1, 2000, pp. 157-175. doi:10.1037/0882-7974.15.1.157

[38] S. Hale, N. S. Rose, J. Myerson, M. J. Strube, M. Sommers, N. Tye-Murray and B. Spehar, "The Structure of Working Memory Abilities across the Adult Life Span," Psychology and Aging, Vol. 26, No. 1, 2011, pp. 92-110. doi:10.1037/a0021483

[39] F. Fiore, E. Borella, I. C. Mammarella and R. De Beni, "Age Differences in Verbal and Visuo-spatial Working 
Memory Updating : Evidence from Analysis of Serial Position Curves," Memory, Vol. 20, No. 1, 2012, pp. 14-27. doi:10.1080/09658211.2011.628320

[40] L. A. Brown, J. R. Brockmole, J. Alan and I. J. Deary, "Processing Speed and Visuospatial Executive Function Predict Visual Working Memory Ability in Older Adults," Experimental Aging Research, Vol. 38, No. 1, 2012, pp. 1-19. doi:10.1080/0361073X.2012.636722

[41] A. Field, "Discovering Statistics Using SPSS (Introducing Statistical Methods S.)," Sage Publications Ltd., London, 2005.

[42] H. Lin, M. Yeh and Y. Kang, "An Investigation of the Relationship between Digit Ratio," Proceedings of the 3rd International Conference on Internationalization, Design and Global Development: Held as Part of HCI International 2009, San Diego, 19-24 July 2009, pp. 266-273.

[43] M. Kozhevnikov, J. Royan, O. Blazhenkova, A. Gorbunov, J. S. Gero and A. K. Goel, "The Role of Immersivity in Three-Dimensional Mental Rotation," In: J. S. Gero and A. K. Goel, Eds., Design Computing and Cognition'08, Springer Netherlands, Dordrecht, 2008, pp. 143-157. doi:10.1007/978-1-4020-8728-8 8

[44] R. Shepard and J. Metzler, "Mental Rotation of ThreeDimensional Objects," Science, Vol. 171, No. 3972, 1971, pp. 701-703. doi:10.1126/science.171.3972.701

[45] B. Sanders, J. R. Wilson and S. G. Vandenberg, "Handedness and Spatial Ability," Cortex, Vol. 18, No. 1, 1982, pp. 79-89. doi:10.1016/S0010-9452(82)80020-8

[46] T. G. Reio, M. Czarnolewski and J. Eliot, "Handedness and Spatial Ability: Differential Patterns of Relationships," Laterality, Vol. 9, No. 3, 2004, pp. 339-358. doi: $10.1080 / 13576500342000220$

[47] J. T. Manning, R. L. Trivers, R. Thornhill and D. Singh, "The 2nd:4th Digit Ratio and Asymmetry of Hand Performance in Jamaican Children," Laterality, Vol. 5, No. 2, 2000, pp. 121-132. doi:10.1080/135765000396744

[48] J. T. Manning and M. Peters, "Digit Ratio (2D:4D) and Hand Preference for Writing in the BBC Internet Study," Laterality, Vol. 14, No. 5, 2009, pp. 528-540. doi: $10.1080 / 13576500802637872$

[49] J. S. Janowsky, B. Chavez, D. Brian and E. Orwoll, "The Cognitive Neuropsychology of Sex Hormones in Men and Women," Developmental Neuropsychology, Vol. 14, No. 2-3, 1998, pp. 421-440. doi: $10.1080 / 87565649809540719$

[50] E. Kemps and R. Newson, "Patterns and Predictors of Adult Age Differences in Mental Imagery," Aging, Neuropsychology, and Cognition, Vol. 12, No. 1, 2005, pp. 99-128. doi:10.1080/13825580590925152

[51] I. E. Dror and S. M. Kosslyn, "Mental Imagery and Aging," Psychology and Aging, Vol. 9, No. 1, 1994, pp. 90102. doi:10.1037/0882-7974.9.1.90 